\title{
Damage Control Surgery for Abdominal Compartment Syndrome Caused by Delayed Rupture of Hepatic Subcapsular Hematoma
}

\author{
Chan Yong Park1, Kwang Hee Yeo', Ho Hyun Kim¹, Seon Hee Kim¹, Hyun Min Cho', \\ Hoon Kwon', Chang Ho Jeon', Chang Won Kim², Seok Ran Yeom ${ }^{3}$ \\ 'Department of Trauma Surgery, Pusan National University Hospital, Busan, Korea \\ ${ }^{2}$ Department of Radiology, Pusan National University Hospital, Busan, Korea \\ ${ }^{3}$ Department of Emergency Medicine, Pusan National University Hospital, Busan, Korea
}

We report a case of damage control surgery (DCS) performed for abdominal compartment syndrome (ACS) due to delayed rupture of hepatic subcapsular hematoma, confirmed by initial abdominal computed tomography (CT) after blunt trauma. A 58-year-old man presented to the emergency department with a Glasgow Coma Scale Score 5 after a passenger traffic accident. Abdominal CT demonstrated a large crescent-shaped hepatic subcapsular hematoma (10 $\times 5 \times 17 \mathrm{~cm}$ in size). During neurosurgery, ACS developed due to delayed rupture of a subcapsular hematoma and DCS was performed. Immediately after temporary abdomen closure (TAC), the patient was transferred to the intervention room and transcatheter arterial embolization (TAE) was performed on the bleeding site from the liver surface. Two days after surgery, gauze removal and definitive closure of the abdomen were performed. The patient was transferred to the rehabilitation department 4 weeks postoperatively.

(Trauma Image Proced 2017(1):17-21)

Key Words: Intra-abdominal Hypertension; Rupture; Hematoma; Liver; Embolization, Therapeutic

\section{CASE}

A 58-year-old man presented to the emergency department with a Glasgow Coma Scale Score 5 after a passenger traffic accident. His vital signs were blood pressure 110/60 $\mathrm{mmHg}$, pulse rate 118 beats $/ \mathrm{min}$, respiration rate 27 breaths $/$ min, body temperature $36.6^{\circ} \mathrm{C}$, and oxygen saturation 99\%. The Injury Severity Score (ISS) was 43, revised trauma score (RTS) was 5.03, and trauma and injury severity score (TRISS) was $15.2 \%$. Laboratory examination revealed white blood cell count
(WBC) $7.52 \times 10^{3} / \mu \mathrm{L}$, hemoglobin $(\mathrm{Hb}) 11.0 \mathrm{~g} / \mathrm{dL}$, platelet $39 \times 10^{3} / \mu /$, lactic acid $3.8 \mathrm{mmol} / \mathrm{L}$, and base excess -6.6. Abdominal computed tomography (CT) demonstrated a large crescent-shaped subcapsular hematoma of the right hepatic lobe (segments 5-8) without a definite parenchymal laceration. The subcapsular hematoma was $10 \times 5 \times 17 \mathrm{~cm}$ in size and was not ruptured. Contrast leakage was observed at the parenchymal boundary (Fig. 1., 2.). The brain showed severe subdural hematoma $(\mathrm{SDH})$, extradural hematoma $(\mathrm{EDH})$, and intracerebral hemorrhage (ICH), and emergency craniotomy

Received: February 4, 2017 Revised: February 25, 2017 Accepted: March 5, 2017

Correspondence to: Chan Yong Park, Department of Trauma Surgery, Pusan National University Hospital, 179, Gudeok-ro, Seo-gu, Busan, Korea

Tel: 82-51-240-7369, Fax: 82-51-240-7719, E-mail: wkafyddl@hanmail.net

Copyright (c) 2017 Korean Association for Research, Procedures and Education on Trauma. All rights reserved.

@) This is an open-access article distributed under the terms of the Creative Commons Attribution Non-Commercial License (http://creativecommons.org/ licenses/by-nc/4.0) which permits unrestricted noncommercial use, distribution, and reproduction in any medium, provided the original work is properly cited 


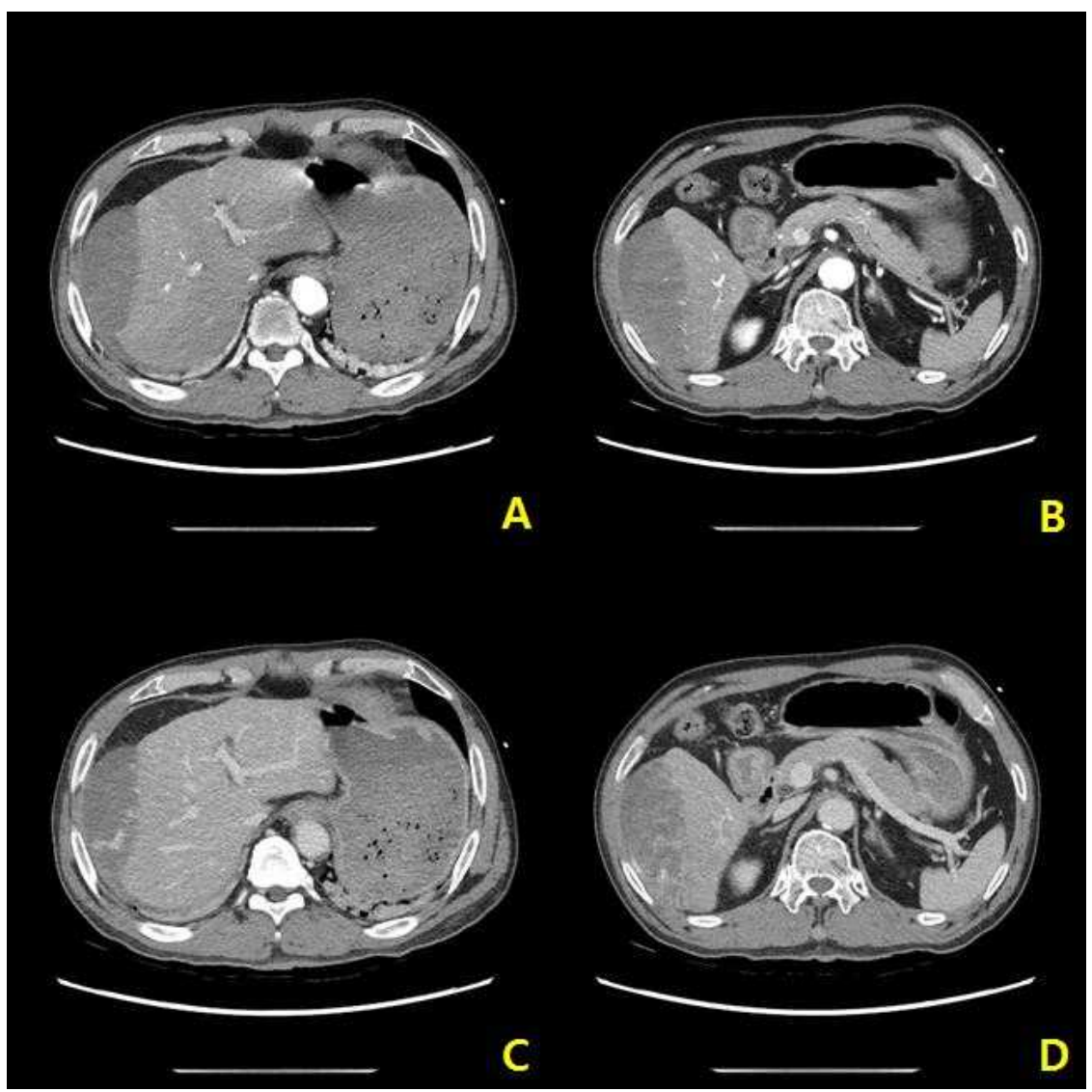

Fig. 1. Abdominal CT (axial view) demonstrates a large crescent-shaped subcapsular hematoma of the right hepatic lobe without a definite parenchymal laceration. The subcapsular hematoma was not ruptured. Contrast leakage was observed at the parenchymal boundary.

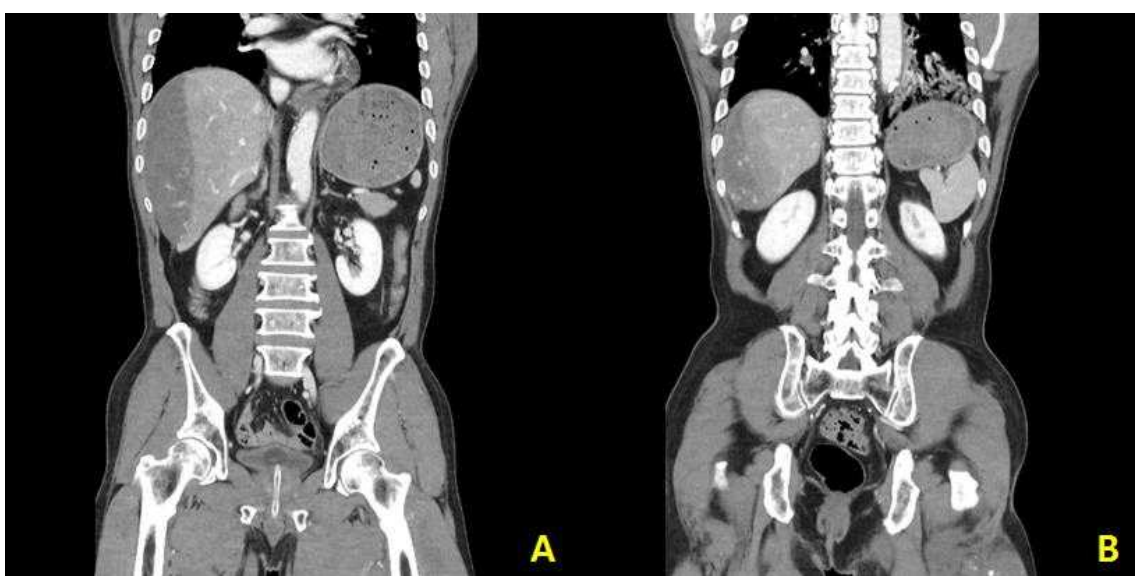

Fig. 2. Abdominal CT (coronal view) demonstrates a large crescent-shaped subcapsular hematoma of the right hepatic lobe without a definite parenchymal laceration. The subcapsular hematoma was not ruptured. Contrast leakage was observed at the parenchymal boundary. 


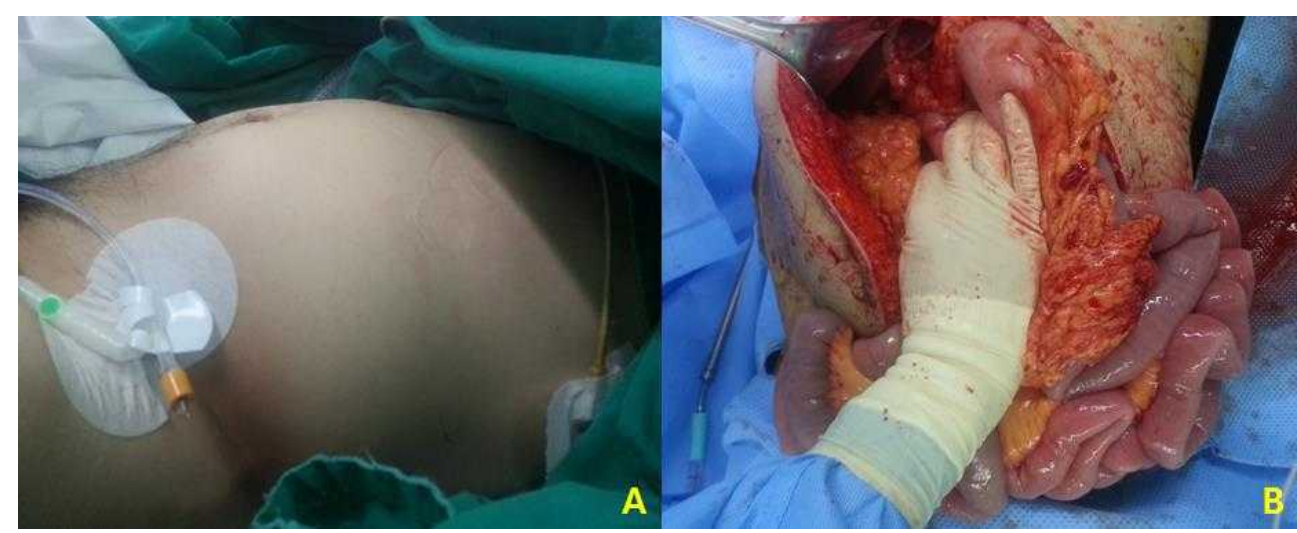

Fig. 3. The abdomen was severely distended during craniotomy (A). A capsule of hepatic subcapsular hematoma was ruptured, causing diffuse bleeding from the liver surface. A severe intestinal edema was also noted (B).

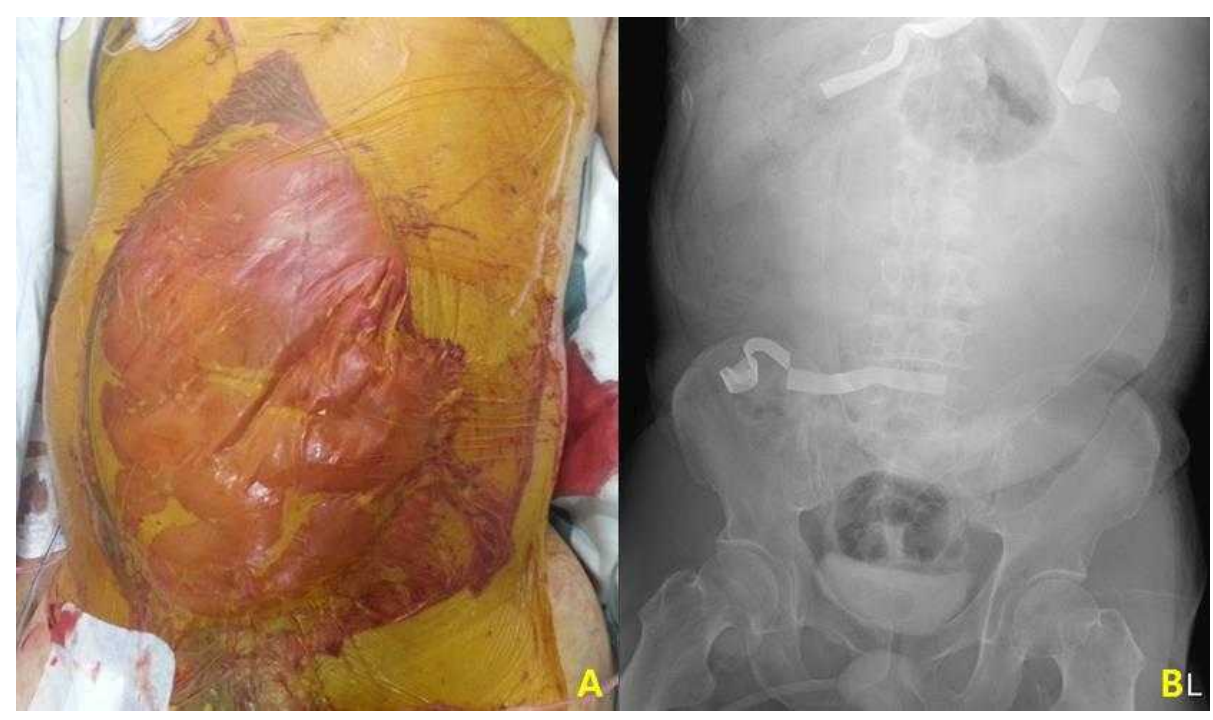

Fig. 4. A perihepatic packing was performed, two 4-layered gauzes were placed between the liver and lateral abdominal wall (one on segments 5 and 6, another on segments 7 and 8) and the abdominal wound was closed temporarily.

was performed. During surgery, systolic blood pressure decreased to less than $90 \mathrm{mmHg}$, heart rate increased to more than 120 beats/min, and abdominal distension became prominent. In the operating room, intra-abdominal ongoing hemorrhage accompanied by abdominal compartment syndrome (ACS) was suspected and emergency laparotomy was performed. A large amount of hematoma of more than $3 \mathrm{~L}$ was observed in the abdominal cavity at laparotomy and 4-quadrant gauze packing was performed. A capsule of hepatic subcapsular hematoma was ruptured, causing ongoing bleeding from the ruptured hematoma. In addition, severe intestinal edema was noted (Fig. 3.). After contacting the interventional radiologist for transcatheter arterial embolization (TAE), we performed damage control surgery (DCS). A perihepatic packing and temporary abdomen closure (TAC) was performed (Fig. 4.). Immediately after the operation, TAE was performed for the bleeding arteries from the ruptured hematoma (Fig. 5.). The patient was admitted to the trauma intensive care unit (TICU) and his 


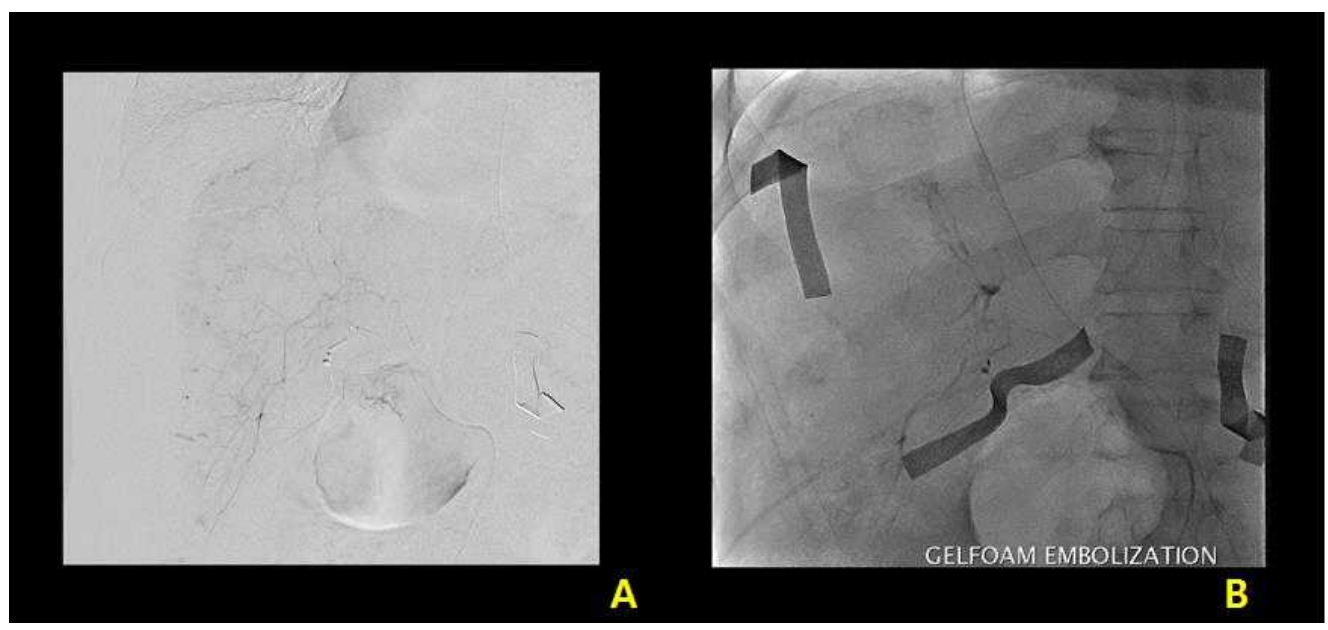

Fig. 5. Immediately after the operation, an embolization was performed for the bleeding arteries from the ruptured hematoma.

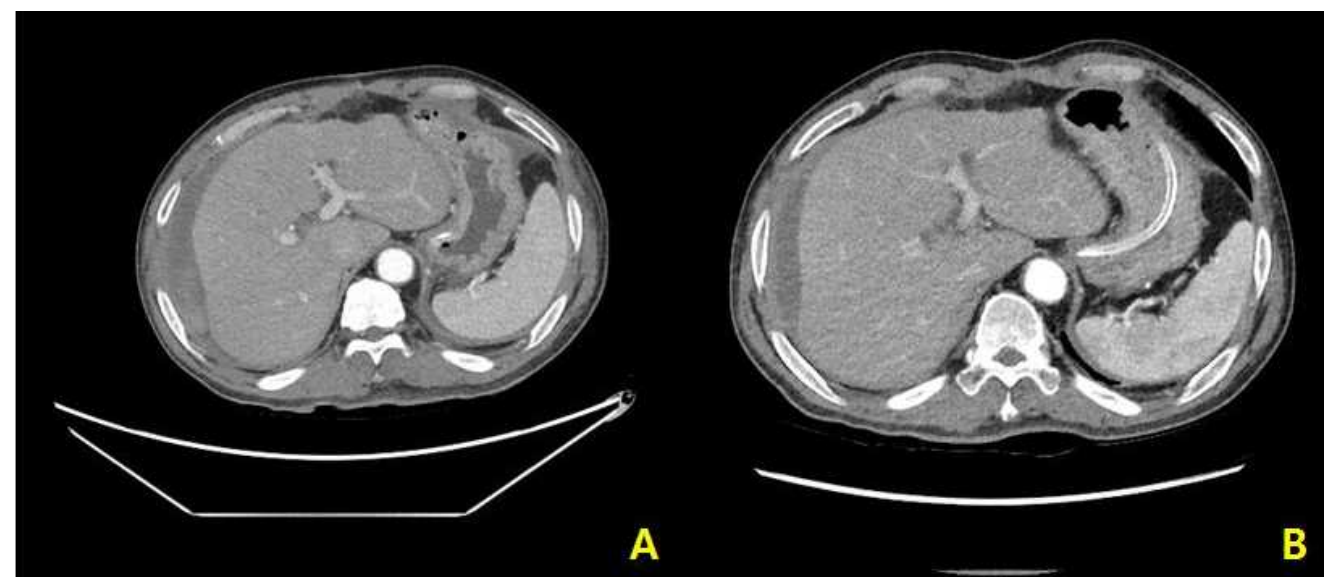

Fig. 6. The ruptured hematoma decreased gradually over time. Abdominal CT was performed $2(A)$ and 5(B) weeks postoperatively.

physiologic status improved. Two days postoperatively, gauze removal and definitive closure of the abdomen were performed. The ruptured hematoma decreased gradually over time (Fig. 6.). The patient was transferred to the rehabilitation department 4 weeks postoperatively.

\section{DISCUSSION}

Hepatic subcapsular hematoma caused by blunt trauma is not a rare condition. However an unruptured large subcapsular hematoma is rare. Taff et al. (1) reported a case of sudden death due to delayed rupture of a hepatic subcapsular hematoma. The possibility of rupture should be considered when the size of the supcapsular hematoma is large as in this case. In patients with large hepatic subcapsular hematoma rupture, if bleeding control is difficult or coagulopathy progresses, TAE after DCS also can be a treatment option $(2,3)$

\section{Conflict of Interest Statement}

This work was supported by clinical research grant from Pusan National University Hospital in 2016.

\section{REFERENCES}

1. Taff ML, Wolodzko AA, Boglioli LR. Sudden death due to 
delayed rupture of hepatic subcapsular hematoma following blunt abdominal trauma. The American journal of forensic medicine and pathology. 1990;11(3):270.

2. Letoublon C, Morra I, Chen Y, Monnin V, Voirin D, Arvieux C. Hepatic arterial embolization in the management of blunt hepatic trauma: indications and complica- tions. Journal of Trauma and Acute Care Surgery. 2011; 70(5):1032-7.

3. Park CI, Lee SB, Yeo KH, Lee S, Park SJ, Kim HH, et al. Successful TAE after DCS for Active Arterial Bleeding from Blunt Hepatic Injury in a Child: A Case Report. Journal of Trauma and Injury. 2016;29(2):47-50. 